\title{
Vacunación para hepatitis B en pacientes adultos infectados con virus de inmunodeficiencia humana
}

\author{
Carlos A. Ramírez O., Daniel G. Fernández A., Sandra L. Valderrama B., \\ Carlos H. Gómez Q., José R. Támara R. y Carlos A. Álvarez M.
}

Pontificia Universidad Javeriana. Bogotá, Colombia (CARO, DGFA, SLVB, CHGQ, JRTR, (AAM) Hospital Universitario San Ignacio, Bogotá (SLVB, CHGQ, JRTR, CAAM).

Recibido: 3 marzo 2008 Aceptado: 23 septiembre 2008

Conflictos de interés. Todos los autores declaran no tener conflictos de interés. Esta revisión de tema no recibió financiamiento externo.

Correspondencia a: Sandra Liliana Valderrama Beltrán sandra.valderrama@gmail.com

\section{Introducción}

$\mathrm{E}$ n pacientes con infección por VIH es relativamente frecuente la infección por el virus de la hepatitis B (VHB) debido a sus rutas compartidas de transmisión. Adicionalmente, la enfermedad hepática debido a infección crónica por el virus B es una causa importante de muerte por causa no asociada al síndrome de inmunodeficiencia adquirida (SIDA) en pacientes infectados con VIH, teniendo mayor riesgo los pacientes con bajos recuentos de CD $4^{1,2}$. Igualmente importante es el hecho que los pacientes coinfectados progresan con mayor frecuencia a cirrosis y carcinoma hepatocelular; esta rápida progresión ha llevado a la actual recomendación de un inicio más temprano de terapia anti-retroviral en este grupo de pacientes $^{3-6}$.

En sujetos inmunocompetentes, la vacunación contra hepatitis B es la medida más efectiva para prevenir la infección y sus consecuencias; en pacientes inmunocomprometidos ocurre lo mismo pero la respuesta observada a la vacunación es inferior, lo cual es particularmente evidente si el estado de inmunosupresión empeora. Se ha observado que la respuesta de anticuerpos frente al antígeno usado en la vacuna contra el virus $\mathrm{B}$ es dependiente de células $\mathrm{T}$ lo cual frecuentemente induce una respuesta de títulos inferior en pacientes infectados con $\mathrm{VIH}^{7,8}$, al igual que una caída más rápida hacia niveles no protectores, comparada con pacientes sin $\mathrm{VIH}^{9}$. Lo anterior hace importante conocer el momento, la dosis y la frecuencia de refuer- zos adecuada que confiablemente nos haga pensar en un acercamiento costo-efectivo en la vacunación de pacientes infectados con VIH.

\section{Epidemiología}

A pesar de ser reconocida desde hace 40 años, la hepatitis B constituye actualmente un problema importante de salud pública ${ }^{10,11}$. Aproximadamente dos billones de personas en el mundo tienen evidencia de infección previa con VHB, más de 350 millones son portadores crónicos del virus y aproximadamente existe un millón de muertes al año debidas a cirrosis y carcinoma hepatocelular ${ }^{12,13}$. La transmisión es principalmente a través de contacto sexual, contacto de secreciones infectadas con mucosas, vía percutánea y transmisión vertical o perinatal. La forma mediante la cual los individuos se infectan varía acorde la edad, hábitos, co-morbilidad y endemia de la zona poblacional. Es así que la infección en áreas endémicas como en África, este de Europa, Asia central, China, sudeste asiático, islas del Pacífico y la Amazonía, ocurre en etapas tempranas de la vida, tal vez originada por transmisión vertical y en la infancia temprana, siendo alrededor de $70 \%$ de la población positiva para infección previa, con alto riesgo de cronicidad por hepatitis $\mathrm{B}^{13,14}$. En contraparte, en regiones con baja a intermedia endemia la mayoría de las infecciones ocurren en adolescentes y adultos jóvenes, principalmente secundarias a transmisión sexual, seguida de la adquisición por la vía percutánea como la segunda 
causa $^{15}$. Debido a las rutas compartidas de transmisión como son el contacto sexual y el uso de drogas intravenosas, se ha observado que 28 a $90 \%$ de los pacientes infectados con VIH tienen marcadores serológicos de contacto previo con VHB (anti-HBs o anti-HBc positivos) y entre 10 y $15 \%$ permanecen crónicamente infectados (HBsAg positivo por más de 6 meses) ${ }^{16,17}$. En un estudio realizado en 16.000 pacientes en diferentes estados de Norteamérica y Puerto Rico, la prevalencia de co-infección hepatitis $\mathrm{B}$ crónica en pacientes infectados con VIH mostró ser de 7,6\%, estudio en el cual también se documentó que la incidencia se incrementó en afroamericanos, alcohólicos, recientes usuarios de drogas intravenosas y pacientes con SIDA ${ }^{18}$. Similarmente, en Tailandia se encontró una prevalencia de $8,7 \%$ (60 de 692 pacientes infectados con VIH que recibían terapia antirretroviral) ${ }^{19,20}$. En Chile, Northland en 1993 documentó positividad para HBsAg en $9 \%$ de 284 pacientes infectados con $\mathrm{VIH}^{21}$. En Colombia, en un estudio realizado por los autores en una cohorte descriptiva de 1.100 pacientes infectados con VIH, se encontró hepatitis B crónica en $6,8 \%$ y presencia de marcadores serológicos de infección antigua en $40 \%{ }^{22}$.

\section{Historia natural y patogénesis}

El VHB no es directamente citopático y de esta manera el pronóstico y la extensión de la infección son principalmente influenciados por la calidad y diversidad de la respuesta inmune ${ }^{23-25}$. Los factores inmunológicos que contribuyen al control de la replicación, excreción y desarrollo de complicaciones asociadas con infección crónica por el VHB como el carcinoma hepatocelular, involucran la activación tanto del sistema humoral como el mediado por células ${ }^{23}$. Así, en pacientes que presentan hepatitis aguda autolimitada tenemos un tipo de respuesta inmune principalmente dirigida contra epítopes de la cápside viral, la polimerasa y proteínas del core, la cual produce la excreción del virus impidiendo la progresión a fase crónica. Esta clase de respuesta es predominantemente de tipo $\mathrm{TH} 1$ e involucra las citoquinas IL2 y $\gamma$ IFN las cuales contribuyen a la injuria del hepatocito durante la fase de recuperación. En contraste, pacientes con hepatitis B crónica muestran tendencia a desarrollar una respuesta humoral de tipo TH2 con producción de IL4, IL5 e IL10 las cuales promueven la producción de anticuerpos en mayor medida que el aclaramiento del virus, produciendo inflamación persistente con mayor riesgo de carcinoma hepatocelular ${ }^{23,26,27}$. Es así como en el contexto de la infección por VIH, debido a la alteración en la inmunidad celular, la progresión hacia hepatitis cró- nica es cinco veces más alta comparada con pacientes $\sin \mathrm{VIH}^{28,29}$. Posterior a la progresión a hepatitis B crónica el curso de la enfermedad puede ser dividido en cuatro fases, inmunotolerante, inmunoactivo, portador inactivo y reactivación.

- La fase inmunotolerante puede durar décadas y se caracteriza por altos niveles de ADN del VHB lo cual refleja alta replicación y mínima actividad inmune, así mismo se observan enzimas hepáticas normales y baja actividad necro-inflamatoria. Esta fase se encuentra en personas que adquieren la enfermedad en el nacimiento o en la infancia temprana, el paciente esta usualmente asintomático y se presenta infrecuentemente en las personas que adquieren la infección en la adolescencia o a posteriori ${ }^{30,31}$.

- La segunda fase o inmunoactiva está caracterizada por elevación de transaminasas, niveles fluctuantes de ADN del VHB y necro-inflamación hepática; puede tener duración variable, desde meses hasta años, lo cual se relaciona con la extensión del daño hepático, pudiendo ser la reactivación lo suficientemente grave como para provocar infección aguda fulminante ${ }^{11}$. En pacientes infectados con VIH en esta etapa, existe una reacción paradójica en la cual existe un menor grado de elevación de transaminasas con mayor grado de progresión a fibrosis, con el consecuente riesgo de cirrosis ${ }^{32}$

- En la tercera fase o de portador inactivo, los niveles de ADN viral disminuyen, ocurriendo seroconversión del antígeno e (HBeAg), con producción de anticuerpos contra el antígeno e (anti-HBe) o desapareciendo su nivel sérico. Igualmente, las transaminasas se normalizan y la patología hepática entra en fase quiescente ${ }^{11}$. La seroconversión en adultos hacia la fase de portador inactivo ocurre a una tasa de 8 a $15 \%$ por año en pacientes no infectados por VIH. En pacientes co-infectados con VIH las tasas de aclaramiento espontáneo del HBsAg y $\mathrm{HBeAg}$ están disminuidas con respecto a pacientes sin infección por VIH. A largo plazo los pacientes infectados con VIH presentan desaparición del HBeAg, 12\% comparado con $49 \%$ de controles sin $\mathrm{VIH}$, en 5 años de seguimiento ${ }^{17}$. En un estudio comparativo entre mujeres embarazadas en Zambia con hepatitis B crónica, aquellas co-infectadas con VIH presentaron niveles de $\mathrm{HBeAg}$ detectables dos veces mayor que el grupo control, hallazgos que pudieran sugerir que la infección por VIH retrasa la transición hacia portador inactivo ${ }^{33}$.

- La cuarta fase es la reactivación de hepatitis B. Puede ocurrir espontáneamente o bajo condiciones de inmunosupresión y está asociada con elevación de ALT y de los niveles de ADN viral; no ocurre en todos los pacientes con hepatitis $\mathrm{B}$ crónica ${ }^{11}$. 
En suma, cabe insistir en que los pacientes con coinfección tienen mayor riesgo de desarrollar cirrosis, carcinoma hepatocelular y mayor mortalidad ${ }^{3,4}$.

\section{Vacunación}

Las vacunas disponibles actualmente para prevenir la infección por el VHB son extremadamente seguras y tienen una eficacia mayor a $90 \%$ en pacientes inmunocompetentes, de tal forma que esta enfermedad podría ser erradicada si se garantizara la vacunación global $^{34}$. Lastimosamente, la vacunación contra el VHB dista de ser universal, por una variedad de razones de índole económica, política y social. Respecto a este último factor se debe tener en cuenta que los factores de riesgo que hacen necesaria la vacunación para hepatitis $\mathrm{B}$ entre adultos (hombres que tienen sexo con hombres, personas con múltiples parejas sexuales, drogadictos que usan jeringas, personas con antecedente de enfermedad de transmisión sexual, entre otras) constituyen prácticas o estilos de vida socialmente estigmatizados, lo cual genera una barrera para acceder a la inmunización. Otro factor en contra de lograr mejores índices de vacunación es la ausencia de la vacuna de VHB en los esquemas de inmunización de algunos países, especialmente naciones en vías de desarrollo, al punto que para el año 2000, solamente 116 de 215 países en el mundo tenía una política de vacunación para todos los recién nacidos ${ }^{35}$. Hoy en día es mundialmente aceptado que la vacunación de los recién nacidos contra VHB, reduce la transmisión posterior en niños y adultos, por lo que debe hacer parte de los programas de inmunización ${ }^{13}$. En Colombia, a partir de 1993, la vacuna de hepatitis B es parte del programa ampliado de inmunizaciones obligatorio, de tal manera que se aplica el esquema a todos los recién nacidos de manera gratuita. Sin embargo, no existen programas de vacunación gratuitos para población adulta de alto riesgo.

Hay oportunidades y circunstancias para realizar vacunación a pacientes en alto riesgo de padecer la infección, como cárceles, centros de rehabilitación de drogadictos y clínicas para atención de enfermedades de transmisión sexual ${ }^{35,36}$, pero no se aprovecha esta oportunidad por parte de los organismos de regulación en salud, al punto que un estudio en E.U.A. muestra que $70 \%$ de las personas recientemente infectadas han perdido una oportunidad de vacunación previa a su diagnóstico ${ }^{37}$. El CDC de Atlanta, E.U.A., recomienda que la vacuna contra el VHB se le debe administrar a todo paciente que consulte por enfermedades de transmisión sexual ${ }^{38}$ por tratarse de patologías que comparten una misma ruta para causar infección.
Tipos de vacunas. La primera vacuna contra el VHB fue autorizada para su uso en E.U.A. en el año $1982^{37}$ $\left(\right.$ Heptavax $^{\circledR}$ B, Merck Sharp \& Dohme). Esta vacuna era preparada por concentración y purificación de plasma de portadores de HBsAg para producir partículas subvirales de $22 \mathrm{~nm}$ que contenían HBsAg únicamente. Esta vacuna ya no es empleada en E.U.A., dado que se consideró un riesgo potencial para transmisión de infecciones por el uso de suero humano, pero aún se emplea en países en vía de desarrollo, por su bajo costo y alta posibilidad de encontrar plasma con HBsAg en zonas endémicas ${ }^{32}$. Este biológico fue reemplazado en E.U.A. por vacunas recombinantes, que ingresaron al mercado norteamericano en 1986 (Engerix-B ${ }^{\circledR}$ de Glaxo SmithKline y Recombivax $\mathrm{HB}^{\circledR}$ de Merck, and Co). Estas vacunas contienen HBsAg no glicosilado, expresados de ADN recombinante de la levadura Saccharomyces cerevisiae. Dicho HBsAg ha sido purificado, de tal forma que el producto final contiene más de $95 \%$ de HBsAg y menos del $5 \%$ de proteínas derivadas de la levadura, sin ADN de ésta detectable $^{39,49}$. El tercer tipo de vacuna contra VHB es la vacuna recombinante derivada de células mamíferas, la cual aún no se encuentra disponible en el mercado. Existen formas combinadas de la vacuna del VHB. En el año 2001, la FDA dio aprobación a la vacuna Twinrix ${ }^{\circledR}$ de Glaxo SmithKline, combinación de las vacunas de hepatitis A y B (contiene HBsAg recombinante y virus de la hepatitis A inactivado $)^{40}$. La vacuna de la hepatitis B también se encuentra disponible en forma combinada con otros biológicos, dirigida a la población pediátrica, como es el caso de Comvax ${ }^{\circledR}$ (Merck and Co) que contiene HBsAg recombinante, Haemophilus influenzae tipo B conjugado y complejo de proteína de membrana externa de Neisseria meningitidis. Otra vacuna es Pediarix ${ }^{\circledR}$, (Glaxo SmithKline), la cual contiene HBsAg, recombinante, toxoide diftérico y toxoide tetánico, poliovirus inactivado y pertusis acelular adsorbida.

Indicaciones actuales para inmunización contra virus de hepatitis $B$

- Todos los niños.

- Todas las personas menores de 18 años.

- Personas con riesgo ocupacional (trabajadores del área de la salud expuestos a sangre o fluidos corporales potencialmente contaminados).

- Pacientes con enfermedad renal crónica terminal, incluyendo aquellos en pre diálisis, hemodiálisis y diálisis peritoneal.

- Contactos sexuales de portadores de VHB.

- Personas que han tenido más de una pareja sexual en seis meses.

- Antecedente de enfermedad de transmisión sexual. 
- Hombres que tienen relaciones sexuales con hombres.

- Personas que se inyectan drogas ilícitas.

- Pacientes con infección por VIH.

- Pacientes con enfermedad hepática crónica.

Eficacia. Una respuesta inmune positiva a la vacunación es definida como la aparición de anti-HBs en un valor mayor a $10 \mathrm{UI} / \mathrm{L}$. Los títulos séricos protectores se desarrollan en 95 a $99 \%$ de los niños, adolescentes y en $90 \%$ de los adultos sanos que reciben una serie de tres dosis intramusculares de la vacuna, pero la respuesta de anti-HBs es reducida en personas sobre 60 años de edad (con respuesta en $75 \%$ de los individuos), en fumadores, alcohólicos, personas con infección por VIH, pacientes en hemodiálisisis ${ }^{41,42}$, enfermedad hepática crónica, viéndose más comprometidos en este último grupo aquellos con hepatopatía grave ${ }^{43}$. Los títulos séricos protectores de anti HBs son directamente proporcionales al número de dosis recibidas. En adolescentes y adultos menores de 40 años, aproximadamente 30 a $55 \%$ de los pacientes adquiere respuesta con anticuerpos protectores después de la primera dosis de la vacuna, $75 \%$ lo hace con la segunda dosis y más del $90 \%$ lo logra después de la tercera dosis. La memoria inmunológica obtenida con la vacuna ha demostrado persistir por al menos 15 a 20 años, no siendo necesario hacer controles periódicos de anti-HBs en pacientes inmunocompetentes, así como tampoco está recomendado aplicar dosis de refuerzo ${ }^{44}$. Es importante conocer que $60 \%$ de las personas vacunadas pierden los anti-HBs detectables, pero no la inmunidad después de finalizar el esquema de vacunación.

Esquema recomendado. La inmunización contra el VHB requiere de tres dosis, administradas por vía intramuscular en el deltoides en los niños y adultos y en el área anterolateral del tercio proximal de la pierna en neonatos (no se recomienda la aplicación a nivel glúteo por producir menor respuesta anti-HBs en los pacientes en quienes se emplea este sitio para administración del biológico ${ }^{45}$. El esquema recomendado por el CDC para vacunación contra VHB en adultos es: $0-1-6$, es decir, una dosis inicial, seguido por una segunda dosis al mes y una tercera dosis a los 6 meses de la dosis inicial. Las vacunas actualmente disponibles para adultos tienen una concentración de $20 \mu \mathrm{g}$ en $1 \mathrm{ml}$ y $10 \mu \mathrm{g}$ en $1 \mathrm{ml}$ para Engerix $\mathrm{B}^{\circledR}$ y Recombivax $\mathrm{HB}^{\circledR}$ respectivamente. Esta última tiene una presentación adicional de $40 \mu \mathrm{g}$ en $1 \mathrm{ml}$ para pacientes en diálisis ${ }^{44}$.

Los esquemas de vacunación actualmente recomendados en adultos se resumen en la Tabla 1. Se puede aplicar un esquema alternativo (igual para am-

\begin{tabular}{|c|c|c|c|c|}
\hline Vacuna & Grupo & $\begin{array}{c}\text { Dosis } \\
(\mu \mathbf{g})\end{array}$ & $\begin{array}{l}\text { Volumen } \\
\qquad(\mathrm{ml})\end{array}$ & $\begin{array}{c}\text { Esquema } \\
\text { (meses) }\end{array}$ \\
\hline Engerix $B^{\circledR}$ & $>20$ años & 20 & 1 & $0-1-6$ \\
\hline \multirow[t]{2}{*}{$($ Amp $\times 20 \mu \mathrm{g})$} & Pacientes en hemodiálisis & 40 & 2 & $0-1$ - 6 ó \\
\hline & & & & $0-1-2-6$ \\
\hline Recombivax $\mathrm{HB}^{\circledast}$ & $>20$ años & 10 & 1 & $0-1-6$ \\
\hline \multirow[t]{2}{*}{$(A m p \times 10 \circ 40 \mu \mathrm{g})$} & Pacientes en hemodiálisis & 40 & 1 & $0-1-6$ ó \\
\hline & & & & $0-1-2-6$ \\
\hline
\end{tabular}

bas presentaciones de la vacuna) para adultos de la siguiente manera:

- 0 - 1 - 4 meses

- 0 - 2 - 4 meses

Pruebas post vacunación. Dado que la vacunación contra VHB tiene una tasa de respuesta de 95\%, las pruebas de anti-HBs de rutina no están recomendadas, salvo en los siguientes casos:

- Trabajadores de la salud.

- Pacientes en diálisis crónica.

- Parejas sexuales de pacientes con HBsAg positivo.

- Pacientes infectados con VIH.

- Otros pacientes inmunocomprometidos.

- Recién nacidos de madres con HBsAg positivo.

En todos estos pacientes se debe realizar la medición de anti-HBs después de uno o dos meses de terminar el esquema de vacunación ${ }^{36,46}$, excepto en los hijos de madres HBsAg positivo, en los que se debe medir los anticuerpos 3 a 12 meses después de finalizar el esquema de vacunación.

Vacunación anti-hepatitis $B$ en pacientes infectados con VIH. Un punto de consenso es que todo paciente con diagnóstico nuevo de infección por VIH debe estudiarse con marcadores para hepatitis $\mathrm{B}^{47,48}$ para descartar infección activa concomitante o estado de portador crónico y evaluar la situación inmunológica contra el VHB, previo al esquema de vacunación. En los pacientes infectados con VIH la efectividad de la vacunación es: $87 \%$ en pacientes con CD4 mayores a 500 céls $/ \mathrm{mm}^{3}$ pero de sólo $33 \%$ en pacientes con CD4 entre 200 y 500 céls $/ \mathrm{mm}^{3}$; adicionalmente los títulos de anticuerpos posteriores a la vacunación son menores $^{49}$ y tienen una menor duración que en las personas sin infección por $\mathrm{VIH}^{50}$. No obstante, los pacientes que logran y mantienen concentraciones plasmáticas suficientes de anti-HBs después de la vacunación tienen protección contra hepatitis $\mathrm{B}$. La respuesta a la 
vacunación está directamente relacionado con el recuento de CD4 de los pacientes, especialmente si el recuento es menor de 500 céls $/ \mathrm{mm}^{3}{ }^{49}$ y parece también existir relación con los niveles de carga viral ${ }^{51}$. Adicionalmente, los pacientes que reciben tratamiento anti-retroviral tienen mejor respuesta a la vacunación que los que no reciben tratamiento ${ }^{48}$. Varios estudios han evaluado el efecto de diversos esquemas de vacunación contra VHB en pacientes infectados con VIH. En algunos se recomienda duplicar la dosis habitual de $20 \mu \mathrm{g}^{52}$ y en otros se recomienda emplear el doble del número de dosis ${ }^{53}$. Incluso un estudio evaluó una ruta distinta de administración de la vacuna, empleando la vía subdérmica ${ }^{54}$, aunque actualmente la recomendación para administración del biológico en todo tipo de pacientes continúa siendo la vía intramuscular. Existen diversos esquemas de vacunación para VHB en pacientes infectados con VIH y se hace necesario la unificación de criterios respecto a este particular. El esquema de vacunación más ampliamente aceptado depende de los CD4 del paciente. En pacientes con CD4 mayores de 500 céls $/ \mathrm{mm}^{3}$ se aplica un esquema de tres dosis $(0,1,6$ meses $)$ de $20 \mu \mathrm{g}$; en pacientes con CD4 entre 200 y 500 céls $/ \mathrm{mm}^{3}$ se aplica un esquema de 4 dosis $(0,1,2,6$ ó 12 meses $)$ de $20 \mu \mathrm{g}$ y en pacientes con CD4 menor de 200 céls $/ \mathrm{mm}^{3}$ no se recomienda realizar vacunación hasta tener idealmente CD4 mayores de 200 céls $/ \mathrm{mm}^{3}{ }^{49,55}$ (Tabla 2).

Los pacientes que presentan HBsAg negativo pero anti-HBc positivo y anti-HBs negativo son infrecuentes entre la población inmunocompetente, pero son frecuentes en la población infectada con VIH. En estos pacientes pueden existir las siguientes condiciones:

- Infección previa con hepatitis B con eliminación de HBsAg, pero incapacidad para mantener o para producir una adecuada respuesta humoral con producción de anti-HBs

- hepatitis B oculta que se define como la presencia de infección con HBsAg negativo pero con carga viral detectable intermitentemente y carga viral positiva en la biopsia hepática; estos pacientes se confirman al encontrar un resultado de carga viral positiva sérica o en la biopsia hepática

\section{Tabla 2. Esquemas de vacunación recomendados en pacientes infectados} con virus de inmunodeficiencia humana

\begin{tabular}{|lccc|}
\hline Pacientes infectados con VIH & Dosis $(\mu \mathbf{g})$ & Esquema & Ref \\
\hline CD4 $>500$ céls $/ \mathrm{mm}^{3}$ & 20 & $0-1-6$ & 49,56 \\
CD4 $200-500$ céls $/ \mathrm{mm}^{3}$ & 20 & $0-1-2-6$ ó 12 & 49,56 \\
CD4 $<200$ céls $/ \mathrm{mm}^{3}$ & & No vacunar hasta & 49,56 \\
& CD4 > 200 céls $/ \mathrm{mm}^{3}$ & \\
\hline
\end{tabular}

- la presencia de co-infección VHB/VHC con disminución de la expresión del primero por predominio del segundo; puede tratarse de un paciente recuperándose de la fase aguda de la enfermedad

- por último, la posibilidad de resultados falsos positivos que son más prevalentes en población de bajo riesgo.

Para definir en cuál de estas situaciones clínicas se encuentra el paciente se recomienda repetir los estudios de anti-HBc. Si los resultados persisten positivos, se recomienda descartar coinfección con VHC. Si no existe coinfección con VHC se recomienda aplicar una dosis de $20 \mu \mathrm{g}$ de vacuna de hepatitis B y medir los niveles de anti-HBs al mes. Si los resultados son positivos se confirma la primera hipótesis. Si los resultados son negativos se debe solicitar una carga viral; si la carga viral es positiva se confirma la segunda hipótesis, si la carga viral es negativa es alta la probabilidad de que el paciente no tenga previo contacto con VHB y se deben completar las tres dosis de vacunación ${ }^{52,56,61}$.

Es importante recordar que en pacientes infectados con VIH se recomienda un control de anti-HBs uno o dos meses después de finalizar la vacunación ${ }^{48,56}$. En pacientes que no respondan al esquema inicial de vacunación se recomienda un esquema de revacunación aumentando la dosis a $40 \mu \mathrm{g}$ y/o aumentar el número de dosis; cualquiera de las dos opciones esta recomendada en la literatura médica ${ }^{53,54}$. Otra posibilidad es utilizar un adyuvante de la respuesta inmune a la vacuna de hepatitis B; para ello, el uso de factor estimulante de colonias granulocito-macrófago ha mostrado ser efectivo mientras que el uso de IL2 no; otro adyuvante conocido es el CPG 7909 un oligo-deoxinucleótido que activa las células dendríticas y células $\mathrm{B}$ vía el receptor Toll-like 9 que ha mostrado mejorar la respuesta a la vacunación. Sin embargo, el uso de estos adyuvantes no se recomienda rutinariamente ${ }^{57,58}$. Para definir que un paciente no ha respondido a la vacunación debe estar en tratamiento anti-retroviral y se le deben aplicar por lo menos tres dosis adicionales de vacuna de hepatitis $\mathrm{B}^{59,60}$.

Es difícil distinguir entre disminución de la inmunidad y ausencia de respuesta en pacientes con título desconocido anti-HBs después de vacunación para hepatitis B. En estos casos se recomienda una dosis de vacunación de $20 \mu \mathrm{g}$ y del primer al tercer mes de vacunación obtener un resultado de anti-HBs. Los no respondedores a la vacuna no lograrán niveles mayores de $10 \mathrm{IU} / \mathrm{ml}$, los respondedores a la vacuna lograrán una sólida respuesta con niveles mayores de 10 $\mathrm{IU} / \mathrm{ml}^{61}$. Por último, en pacientes infectados con VIH se recomienda un control anual de anti-HBs después de verificar respuesta a la vacunación y si los niveles de 
anti-HBs son menores de $10 \mathrm{IU} / 1$ se recomienda un refuerzo de la vacuna de hepatitis $\mathrm{B}^{61}$.

Efectos adversos. La vacuna contra VHB es generalmente bien tolerada por la mayoría de las personas inmunizadas, reportándose como únicos efectos adversos dolor en el sitio de aplicación y fiebre de bajo grado en un muy pequeño número de $\operatorname{casos}^{43}$. La incidencia de anafilaxia con la aplicación de esta vacuna entre niños y adolescentes es de un caso por 1,1 millones de dosis aplicadas ${ }^{62}$. Con la vacuna derivada de plasma se reportaron algunos casos de síndrome de Guillain Barré que inicialmente se asociaron con la vacunación. Posteriormente se realizó una revisión por parte del Instituto de Medicina de Estados Unidos en el que se concluyó que no existe evidencia suficiente para establecer una relación de asociación entre la vacunación contra VHB actual y síndrome de Guillain Barré $^{63}$.

En pacientes con infección por VIH después de la vacunación se han descrito elevaciones transitorias de carga viral durante el tratamiento anti-retroviral que pueden prolongarse por días o semanas; no se han descrito elevaciones sostenidas de carga viral, cambios en el recuento de CD4 o en la progresión de la infección ${ }^{49}$.

\section{Conclusiones}

La prevención de hepatitis $B$ a través de la vacunación es una importante intervención en salud pública en la población general y especialmente entre las personas infectadas por VIH en quienes adquiere matices especiales por una disminución en esta población en la capacidad de elaborar anticuerpos protectores posteriores a la inmunización.
Los autores recomendamos vacunar a todos los pacientes infectados con VIH sin contacto previo con VHB con recuentos de CD4 mayores a 500 céls $/ \mathrm{mm}^{3}$ con el esquema utilizado en pacientes inmunocompetentes (dosis $20 \mu \mathrm{g}$, esquema $0,1,6$ meses) y con el esquema de 4 dosis $(0,1,2,12$ meses) de $20 \mu \mathrm{g}$ a pacientes con CD4 entre 200 y 500 céls $/ \mathrm{mm}^{3}$ y con posterioridad a la vacunación medir los anticuerpos (anti-HBs) para verificar la respuesta. Si la respuesta a la vacunación no existe al finalizar el esquema se le debe ofrecer un esquema de revacunación. En pacientes con CD4 menores de 200 céls $/ \mathrm{mm}^{3}$ recomendamos no vacunar hasta lograr un aumento en los niveles de CD4 por encima de esta cifra.

No tenemos datos sobre efectividad de la vacunación para VHB en pacientes infectados con VIH en Latinoamérica, este aspecto constituye una línea de investigación de la que se puede obtener valiosa información en beneficio de nuestros pacientes.

\section{Resumen}

La infección por virus de hepatitis B (VHB) representa un problema de salud pública a nivel mundial, especialmente entre las personas infectadas con el $\mathrm{VIH}$, en quienes la coinfección modifica el curso y la historia natural de la enfermedad, así como la morbimortalidad, lo cual hace imperativo diseñar estrategias encaminadas a la prevención de la infección por VHB en pacientes infectados con VIH. La vacunación constituye la forma más importante de prevención, aunque su efectividad se ve alterada, de acuerdo al estado inmunológico y virológico del paciente.

\section{Referencias}

1.- Weber R, Sabin C A, Friis-Moller N, Reiss P, El-Sadr W M, Kirk O, et al. Liver-related deaths in persons infected with the human immunodeficiency virus: the D:A:D study. Arch Intern Med 2006 Aug 14-28; 166: 1632-41.

2.- Bica I, McGovern B, Dhar R, Stone D, McGowan K, Scheib R, et al. Increasing mortality due to endstage liver disease in patients with human immunodeficiency virus infection. Clin Infect Dis 2001; 32 : 492-7.

3.- Bonacini M, Louie S, Bzowej N, Wohl A R. Survival in patients with HIV infection and viral hepatitis B or C: A cohort study. AIDS 2004; 18: 2039-45.
4.- Benhamou Y. Antiretroviral therapy and HIV/hepatitis B virus coinfection. Clin Infect Dis 2004; 38: Suppl 2: S98-S103.

5.- Sulkowski M S. Drug-induced liver injury associated with antiretroviral therapy that includes HIV-1 protease inhibitors. Clin Infect Dis 2004; 38: S90-S97.

6.- Hammer S M, Eron J J Jr, Reiss P, Schooley R T, Thompson M A, Walmsley $\mathrm{S}$, et al; International AIDS Society-USA. Antiretroviral treatment of adult HIV infection: 2008 recommendations of the International AIDS Society-USA panel. JAMA 2008 Aug 6; 300: 555-70.

7.- Wong E K, Bodsworth N J, Slade M A, Mulhall B P, Donovan B. Response to hepatitis $\mathrm{B}$ vaccination in a primary care setting: influence of HIV infection, CD4+ lymphocyte count and vaccination schedule. Int J STD AIDS 1996; 7: 490-4.

8.- Collier A C, Corey L, Murphy V L, Handsfield H H. Antibody to human immunodeficiency virus (HIV) and suboptimal response to hepatitis B vaccination. Ann Intern Med 1988; 109: 101-5.

9.- Biggar R J, Goedert J, Hoofnagle J. Accelerated loss of antibody to hepatitis B surface antigen among immunodeficient homosexual men infected with HIV. N Engl J Med 1987; 316: 630-1.

10.- Blumberg B S, Alter H J, Visnich S. A "new" antigen in leukemia sera. JAMA 1984; 191: 547-9. 
11.- Tran T, Martin P. Hepatitis B: epidemiology and natural history. Clin Liver Dis 2004; 8: 255-66.

12.- Lee W. Hepatitis B virus infection. N Engl J Med 1997; 337: 1733-45.

13.- Poland G, Jacobson R. Prevention of hepatitis B with the hepatitis B vaccine. N Engl J Med 2004; 351: 2832-8.

14.- Hoffmann C, Thio C. Clinical implications of HIV and hepatitis B co-infection in Asia and Africa. Lancet Infect Dis 2007; 7: 402-9.

15.- Edmunds W, Medley G, Nokes D, O'Callaghan C, Whittle H, Hall A. Epidemiological patterns of hepatitis B virus in highly endemic areas. Epidemiol Infect 1996; 117: 313-25.

16.- Gilson R J, Hawkins A E, Beecham M R, Ross E, Waite J, Briggs $\mathrm{M}$, et al. Interactions between HIV and hepatitis B virus in homosexual men: Effects on the natural history of infection. AIDS 1997; 11: 597606.

17.- Rodríguez-Méndez M L, González-Quintela A, Aguilera A, Barrio E. Prevalence, patterns, and course of past hepatitis B virus infection in intravenous drug users with HIV-1 infection. Am J Gastroenterology 2000; 95: 1316-22.

18.- Kellerman S E, Hanson D L, McNaghten A D, Fleming P L. Prevalence of chronic hepatitis B and incidence of acute hepatitits $\mathrm{B}$ infection in human immunodeficiency virus-infected subjects. J Infect Dis 2003; 188: $571-7$.

19.- Merican I, Guan R, Amarapuka D, Alexander M J, Chutaputti A, Chien R N, et al. Chronic hepatitis B virus infection in Asian countries. J Gastroenterol Hepatol 2000; 15: 1356-61.

20.- Law W P, Duncombe C J, Mahanontharit A, Boyd M A, Ruxrungtham K, Lange J M, et al. Impact of viral hepatitis co-infection on response to antiretroviral therapy and HIV disease progression in the HIV-NAT cohort. AIDS 2004; 18: 1169-77.

21.- Zunino E. Epidemiología de la hepatitis B en Chile y esquemas de vacunación en Latinoamérica. Rev Chil Infect 2002; 19: 140-55.

22.- Valderrama S, Vesga J, Munevar M, Grimaldo E, Álvarez C, Gómez C, et al. Descripción de los pacientes con virus de inmunodeficiencia humana y hepatitis B del programa de VIH del Hospital Universitario San Ignacio (HUSI). 2002-2007. Resumen C43. Infectio 2008; 12: S61-S62.

23.- Bertoletti A, Ferrari C. Kinetics of the immune response during $\mathrm{HBV}$ and $\mathrm{HCV}$ infection. Hepatology 2003; 38: 4-13.

24.- Jung M, Pape G. Immunology of hepatitis B infection. Lancet Infect Dis 2002; 2: 43-50.
25.- Lee J, Locarnini S. Hepatitis B virus: pathogenesis, viral intermediates, and viral replication. Clin Liver Dis 8 (2004) 301-20.

26. - Barnaba V, Franco A, Alberti A, Balsano C, Benvenuto R, Balsano F. Recognition of hepatitis $\mathrm{B}$ virus envelope proteins by liverinfiltrating $\mathrm{T}$ lymphocytes in chronic $\mathrm{HBV}$ infection. J Immunol 1989; 143: 2650-5.

27. - Löhr H F, Gerken G, Schlicht H J, Meryer zum Büschenfelde $\mathrm{K} \mathrm{H}$, Fleischer B. Low frequency of cytotoxic liver-infiltrating $\mathrm{T}$ lymphocytes specific for endogenous processed surface and core proteins in chronic hepatitis B. J Infect Dis 1993; 68: 1133-9.

28.- Bodsworth N J, Cooper D A, Donovan B. The influence of human immunodeficiency virus type 1 infection on the development of the hepatitis B carrier state. J Infect Dis 1991; 163: 1138-40.

29.- Shukla N B, Poles M A. Hepatitis B virus infection: co-infection with hepatitis $\mathrm{C}$ virus, hepatitis D virus, and human immunodeficiency virus. Clin Liver Dis 2004; 8: 445-60.

30.- Chang M H. The natural history of hepatitis $B$ virus infection in children. $J$ Gastroenterol Hepatol 2000; 15 (Suppl): E16-9.

31.- Valderrama SL, Cuervo S, Cortes J. Hepatitis $\mathrm{B}$ crónica. Artículo de revisión. Infectio 2007; 11: 201-10.

32.- Colin J F, Cazals-Hatem D, Loriot M A, Martinot-Peignoux M, Pham B N, Auperin A, et al. Influence of human immunodeficiency virus infection on chronic hepatitis B in homosexual men. Hepatology 1999; 29: 1306-10.

33. - Oshitani H, Kasolo F C, Mpabalwani M, Mizuta K, Luo N P, Suzuki H, et al. Prevalence of hepatitis B antigens in human immunodeficiency virus type 1 seropositive and seronegative pregnant women in Zambia. Trans R Soc Trop Med Hyg 1996; 90: 235-36.

34.- Teo E K, Lok A S. Hepatitis B virus vaccination. Uptodate 2007. http:/www. utdol.com/utd/content/topic.do?topicKey= heptitis/15188\&view=print. (Accedido 15 diciembre 2007).

35.- Poland G A. Evaluating existing recommendations for hepatitis $\mathrm{A}$ and $\mathrm{B}$ vaccination. Am J Med 2005; 118: 16s-20s.

36.- Hershey J H, Schowalter L, Bailey S B. Public health perspective on vaccine preventable hepatitis: interesting hepatitis A and $\mathrm{B}$ vaccines into public health settings. Am J Med 2005; 118: 100s-108s.

37. - Goldstein S T, Alter M J, Williams I T, Moyer L A, Judson F N, Mottram K, et al. Incidence and risk factors for acute hepatitis B in the United States, 1982-1998: Implications for vaccination programs. J
Infect Dis 2002; 185: 713-9

38. - Handsfield H H. Hepatitis A and B immunization in persons being evaluated for sexually transmitted diseases. Am J Med 2005; 118: 69s-74s

39.- Davis J P. Experience with hepatitis A and B vaccines. Am J Med 2005; 118: 7s-15s

40.- Anonimous. Notice to Readers: FDA approval of an alternate dosing schedule for a combined hepatitis $A$ and $B$ vaccine $\left(\right.$ Twinrix $\left.^{\circledR}\right)$. MMWR Morb Mortal Wkly Rep 2007; 56: 1057. http:/www.cdc.gov/mmwr/ $\mathrm{PDF} / \mathrm{rr} / \mathrm{rr} 5516 . p d f$. (Accedido 16 octubre 2007).

41.- Lemon S M, Thomas D L. Vaccines to prevent viral hepatitis. N Eng J Med 1997; 336: 196.

42.- Sjogren M H. Prevention of hepatitis B in nonresponders to initial hepatitis $\mathrm{B}$ virus vaccination. Am J Med 2005; 118 (10A): 34s-9s.

43.- Keeffe E B. Acute hepatitis A and B in patients with chronic liver disease: prevention through vaccination. Am J Med 2005; 118: 21s-27s.

44.- Sexually transmitted diseases-Treatment guidelines 2006. Centers for Disease Control and Prevention. http://www.cdc.gov/std/ treatment/2006/hepatitis-b.htm\#hepb3 (Accedido 15 julio 2008).

45.- Hepatitis B vaccine: Drug Information. Uptodate 2007. http://www.utdol.com/utd/ content/topic.do?topicKey=drug_a_k/ $122311 \&$ view $=$ print. (Accedido 15 enero 2008).

46.- Hepatitis B. Pinkbook. http:/www.cdc.gov/ vaccines/pubs/pinkbook/downloads/hepb.pdf (Accedido 15 agosto 2008).

47.- Laurence J. Hepatitis A and B in immunizations of individual infected with human immunodeficiency virus. Am J Med 2005; 118: 75s-83s.

48.- Soriano V, Puoti M, Peters M, Benhamou Y, Sulkowski M, Zoulim F, et al. Care of HIV patients with chronic hepatitis B: update recomendations from the HIV-hepatitis B virus international panel. AIDS 2008; 22: 1139-410.

49.- McGovern B H, Sherman K E. Treatment and prevention of hepatitis $\mathrm{B}$ in the HIV infected patient. Uptodate 2007. http:// www.utdol.com /utd/content/topic.do? topicKey=heptitis $/ 19871 \&$ view $=$ print. (Accedido 1 agosto 2008).

50.- Welch K, Morse A. Improving screening and vaccination for hepatitis $B$ in patients coinfected with HIV and hepatitis C. Am J Gastroenterol 2002; 97: 2928-9.

51.- Koziel M J, Peters M G. Viral hepatitis in HIV infection. N Eng J Med 2007; 356: 1445-54.

52.- Fonseca M O, Pang L W, de Paula 
Cavalheiro N, Barone A A, Lopes M H. Randomized trial of recombinant hepatitis $\mathrm{B}$ vaccine in HIV infected adult patients comparing a standard dose to a double dose. Vaccine 2005; 23: 2902-8

53.- Rey D, Krantz V, Partisani M, Schmitt M P, Meyer P, Libbrecht E, et al. Increasing the number of hepatitis $B$ vaccine injections augments antiHBs response rate in HIV infected patients, effects on HIV-1 viral load. Vaccine 2000; 18: 1161-5.

54.- Ristola M A, Vuola J M, Valle M, Von Reyn F. Antibody responses to intradermal recombinant hepatitis B inmunization among HIV positive subjects. Vaccine 2004; 23: 205-9.

55.- Soriano V, Puoti M, Bonacini M, BrooK G, Cargnel A, Rockstroh J, et al. Care of patients with chronic hepatitis B and HIV co-infection: recommendations from an HIV-HBV International Panel AIDS 2005;
19: 221-40.

56.- Gandhi R, Wurcel A, Lee H, Mc Govern B, Shopis J, Geary M, et al. Response to hepatitis B vaccine in HIV-1-positive subjects who test positive for isolated antibody to hepatitis B core antigen: implications for hepatitis $\mathrm{B}$ vaccine strategies. J Infect Dis 2005; 191: 1435-41.

57.- Brook G. Prevention of viral hepatitis in HIV coinfection. J Hepatol 2006; 44 (Suppl): S104-7.

58.- Cooper C L, Angel J B, Seguin I, Davis H L, Cameron D W. CPG 7909 adjuvant plus hepatitis $\mathrm{B}$ virus vaccination in HIVinfected adults achieves long-term seroprotection for up to 5 years. Clin Infect Dis 2008; 46: 1310-4.

59.- Spach D H. Immunizations for HIV-infected adults: indications, timing, and response. Top HIV Med 2006; 14: 154-8.

60.- Rivas P, Herrero M D, Puente S, Ramírez-
Olivencia G, Soriano V. Immunizations in HIV-infected adults. AIDS Rev 2007; 9: 173-87.

61.- Tedaldi E, Baker R, Moorman A, Wood K, Fuhrer J, McCabe R, et al. Hepatitis A and B vaccination practices for ambulatory patients infected with HIV. Clin Infect Dis 2004; 38: 1478-84.

62.- A comprehensive immunization strategy to eliminate transmission of hepatitis B virus infection in the United States.

Recommendations of the Advisory Committee on Immunization Practices (ACIP) Part II: Immunization of Adults. http://www.cdc.gov/mmwr/PDF/rr/ rr5516.pdf (Accedido 15 enero 2008).

63.- Stratton K R, Howe C J, Johnston R B. Adverse events associated with childhood vaccines other than pertussis and rubella. Summary of a report from the Institute of Medicine. JAMA 1994; 271: 1602-5. 